\title{
Low-Noise 0.8-0.96- and 0.96-1.12-THz Superconductor-Insulator-Superconductor Mixers for the Herschel Space Observatory
}

\author{
Brian D. Jackson, Gert de Lange, Tony Zijlstra, Matthias Kroug, Jacob W. Kooi, Jeffrey A. Stern, and \\ Teun M. Klapwijk
}

\begin{abstract}
Heterodyne mixers incorporating Nb SIS junctions and $\mathrm{NbTiN}-\mathrm{SiO}_{2}-\mathrm{Al}$ microstrip tuning circuits offer the lowest reported receiver noise temperatures to date in the $0.8-0.96-$ and $0.96-1.12-\mathrm{THz}$ frequency bands. In particular, improvements in the quality of the NbTiN ground plane of the SIS devices' on-chip microstrip tuning circuits have yielded significant improvements in the sensitivity of the $0.96-1.12-\mathrm{THz}$ mixers relative to previously presented results. Additionally, an optimized RF design incorporating a reduced-height waveguide and suspended stripline $\mathbf{R F}$ choke filter offers significantly larger operating bandwidths than were obtained with mixers that incorporated full-height waveguides near $1 \mathrm{THz}$. Finally, the impact of junction current density and quality on the performance of the $0.8-0.96-\mathrm{THz}$ mixers is discussed and compared with measured mixer sensitivities, as are the relative sensitivities of the $0.8-0.96-$ and $0.96-1.12-T H z$ mixers.
\end{abstract}

Index Terms-Astronomical satellites, niobium, niobium compounds, radio astronomy, submillimeter-wave mixers, superconductor-insulator-superconductor (SIS) mixers.

\section{INTRODUCTION}

$\mathbf{T}$ HE heterodyne instrument for the far-infrared (HIFI) [1], ${ }^{1}$ is a high-sensitivity, high-resolution heterodyne spectrometer that is being built for the European Space Agency's Herschel Space Observatory [2]..$^{2}$ The instrument's 0.48-1.25- and 1.41-1.91-THz frequency coverage will offer astronomers an unprecedented opportunity to observe a significant fraction of

Manuscript received February 4, 2005; revised July 13, 2005. This work was supported in part by the Technologiestichting STW (of The Netherlands), by the Nederlandse Organizatie voor Wetenschappelijk Onderzoek, and by the European Space Agency under the European Space Research and Technology Centre Research Contract 11653/95.

B. D. Jackson is with the SRON National Institute for Space Research, 9747 AD Groningen, The Netherlands and also with the Kavli Institute of Nanoscience, Faculty of Applied Sciences, Delft University of Technology, 2628 CJ Delft, The Netherlands (e-mail: B.D.Jackson@sron.rug.nl).

G. de Lange is with the SRON National Institute for Space Research, 9747 AD Groningen, The Netherlands (e-mail: G.de.Lange@ sron.rug.nl).

T. Zijlstra, M. Kroug, and T. M. Klapwijk are with the Faculty of Applied Sciences, Kavli Institute of Nanoscience, Delft University of Technology, 2628 CJ Delft, The Netherlands (e-mail: zijlstra@dimes.tudelft.nl; kroug@dimes.tudelft.nl; t.m.klapwijk@tnw.tudelft.nl).

J. W. Kooi is with the California Institute of Technology, Pasadena, CA 91125 USA (e-mail: kooi@submm.caltech.edu).

J. A. Stern is with the Jet Propulsion Laboratory, California Institute of Technology, Pasadena, CA 91109 USA (e-mail: Jeffrey.Stern@jpl.nasa.gov).

Digital Object Identifier 10.1109/TMTT.2005.862717

${ }^{1}$ [Online]. Available: www.sron.nl/divisions/lea/hifi

${ }^{2}$ [Online]. Available: http://www.rssd.esa.int/herschel the submillimeter and far-infrared spectrum, much of which is not observable from ground-based telescopes due to absorption by atmospheric water vapor. In order to take full advantage of this space-based observatory, the HIFI instrument will incorporate state-of-the-art cryogenic heterodyne mixers, with five superconductor-insulator-superconductor (SIS) mixers covering the $0.48-1.25-\mathrm{THz}$ band and two hot-electron bolometer (HEB) mixers covering 1.41-1.91 THz.

Unfortunately, although traditional $\mathrm{Nb}$ SIS mixers offer quantum-limited sensitivities below $0.7 \mathrm{THz}$ [3]-[5], increasing resistive losses in their Nb-based microstrip transmission lines at frequencies above the "gap frequency" of Niobium $\left(F_{\text {gap }, \mathrm{Nb}}=2 \Delta_{\mathrm{Nb}} / h \sim 0.7 \mathrm{THz}\right)$ [6] cause the sensitivities of these mixers to drop significantly above $0.7 \mathrm{THz}$ [7]. However, previous work has shown that the use of $\mathrm{NbTiN}$-based ${ }^{3}$ microstrip RF tuning circuits allows the sensitivity of $0.75-1.0-\mathrm{THz}$ SIS mixers to be significantly improved [8]-[10]. Furthermore, as is discussed in more detail in [11], the integration of $\mathrm{Nb}$ SIS junctions with a $\mathrm{NbTiN}-\mathrm{SiO}_{2}-\mathrm{Al}$ microstrip tuning circuit in which the NbTiN ground plane is deposited at $400{ }^{\circ} \mathrm{C}$ (in place of the room-temperature-deposited films used previously) allows low-noise SIS mixer operation to be extended to at least $1.12 \mathrm{THz}$.

Following from these advances, this paper describes the RF design and performance of the 0.8-0.96- and $0.96-1.12-\mathrm{THz}$ mixers that have been developed for use in Bands 3 and 4 of the HIFI instrument. In particular, this paper discusses the integrated designs of reduced-height waveguide embedding circuits and two-junction SIS tuning circuits that yield efficient coupling of radiation to the mixers' SIS junctions over each frequency band. Additionally, the impact of the current densities and leakage currents of the SIS junctions on their mixing performance is discussed in light of simulations of the heterodyne performance of the mixers' two-junction tuning circuits. This mixer model is also used to compare the relative performance of the 0.8-0.96- and 0.96-1.12-THz mixers.

The optomechanical designs of these mixers (including their corrugated horns), in addition to the designs of their intermediate frequency (IF) output coupling circuits and their shielding from external electromagnetic interference, are described elsewhere [12].

\footnotetext{
${ }^{3}$ For simplicity, the compound $\mathrm{Nb}_{1-x} \mathrm{Ti}_{x} \mathrm{~N}_{1-\delta}$, where $x=0.3$ and $\delta \approx 0$, is referred to as NbTiN throughout this paper.
} 


\section{MiXER AND SIS DEVICE DESIGN}

Previous reports of the development of NbTiN-based SIS mixers made use of two basic mixer geometries: a quasi-optical mixer incorporating a $0.95-\mathrm{THz}$ twin-slot antenna (see [9]) and a waveguide mixer incorporating a full-height $1-\mathrm{THz}$ waveguide (see [10]). These results demonstrated that a $\mathrm{NbTiN}-\mathrm{SiO}_{2}-\mathrm{Al}$ microstrip RF matching network can be integrated with "standard" $1-\mu \mathrm{m}^{2} \mathrm{Nb}-\mathrm{Al}-\mathrm{AlO}_{\mathrm{x}}-\mathrm{Nb}$ SIS junctions to yield low receiver noise temperatures up to $1 \mathrm{THz}$ (and [11] demonstrates that this region of low-noise operation may be extended to at least $1.12 \mathrm{THz}$ by the use of an NbTiN ground plane that is deposited at $400{ }^{\circ} \mathrm{C}$ ). However, the fixed-tuned $\mathrm{RF}$ bandwidth of the 1-THz waveguide mixer in [10] was limited to $\sim 100 \mathrm{GHz}$, whereas the quasi-optical mixer in [10] offered fixed-tuned bandwidths of $\sim 200 \mathrm{GHz}$. This limitation of the previously demonstrated waveguide mixer has been addressed by a redesign of the waveguide embedding geometry [13] and the SIS device's on-chip microstrip tuning circuit to optimize the coupling to the SIS junctions.

The starting point of this redesign (see [14]) was a move from the full-height $1-\mathrm{THz}$ waveguide geometry that was used in [10] to scaled versions of the $0.65-\mathrm{THz}$ half-height waveguide geometry that was used in mixers produced for the James Clerk Maxwell Telescope in Hawaii [15], [16]. In particular, for each of these designs, Fig. 1(a) presents the effective source impedance at the input to the on-chip microstrip tuning circuit that is produced by a combination of the waveguide, the fixed-depth waveguide backshort, the fused quartz substrate in the substrate channel (including the RF choke filter on the substrate), and the "across-the-waveguide" bowtie probe. (This "source impedance" has been calculated in a three-dimensional (3-D) electromagnetic field simulator. ${ }^{4}$ ) From this plot, it is seen that the original $1-\mathrm{THz}$ waveguide design is characterized by a source impedance with a strong frequency dependence and a large imaginary component. In comparison, the source impedances of the 0.65 - and $0.88-\mathrm{THz}$ half-height waveguide designs are much less frequency dependent (although they retain significant reactive components). These results are consistent with those obtained previously in [17] and [18], both in theoretical calculations and in experiment.

As was also demonstrated in [18], further "improvements" in the source impedance offered by the waveguide embedding design are obtained by suspending the SIS device substrate in the substrate channel (and fine-tuning the dimensions in the RF choke filter to recenter the passband of the filter on the center frequency of the design). As is seen in Fig. 1(b), this further reduces the frequency dependence and reactance of the source impedance at the input of the on-chip tuning circuit. (For clarity, only the results for the $0.88-\mathrm{THz}$ design are shown here-the 1.04-THz design is a scaled version of the $0.88-\mathrm{THz}$ design.)

Fig. 2 illustrates the geometry of the $0.88-\mathrm{THz}$ suspended substrate waveguide geometry that is used in the devices described here. The critical waveguide and device substrate dimensions in this design are summarized in Table I. Three-dimensional electromagnetic simulations of the embedding impedance offered by the waveguide geometries are calculated

\footnotetext{
${ }^{4}$ Ansoft Corporation, Pittsburgh, PA.
}

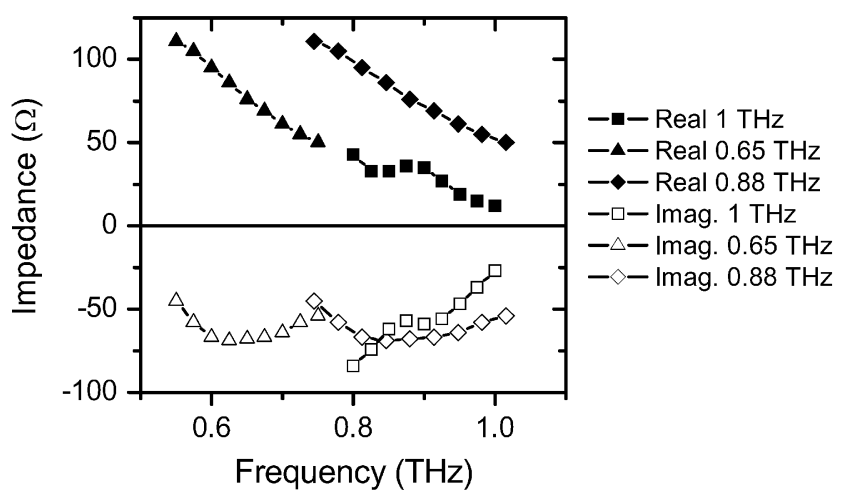

(a)

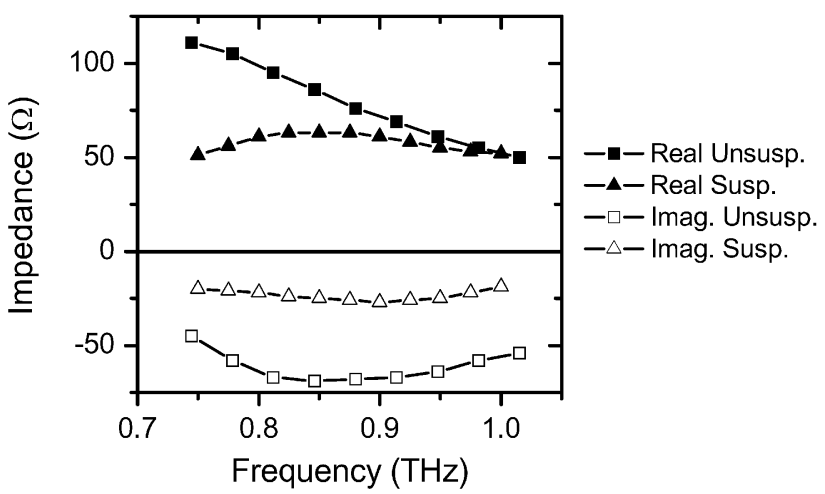

(b)

Fig. 1. Effective source impedance at the input to the on-chip microstrip tuning circuit (at the center of the "across-the-waveguide" bowtie probe, given the fixed-depth waveguide backshort, the fused quartz substrate in the substrate channel, and the RF choke filter patterned on the substrate surface). (a) Comparison of the full-height $1-\mathrm{THz}$ waveguide geometry and the half-height $0.65-\mathrm{THz}$ waveguide geometries used previously with the "unsuspended" half-height $0.88-\mathrm{THz}$ design. (b) Comparison of half-height $0.88-\mathrm{THz}$ waveguide geometries with suspended and unsuspended quartz substrates in the substrate channel. The geometries of the mixers analyzed here are summarized in Fig. 2 and Table I.

for a reference plane at the center of the bowtie waveguide probe (i.e., at the input to the on-chip microstrip tuning circuit). The 1.04-THz mixer design that is used here is a scaled version of the $0.88-\mathrm{THz}$ design.

Finally, the RF designs of the mixers were completed by optimizing the geometry of the twin-junction tuning circuit used in [10] (see Fig. 3) to maximize the coupling of incident RF power to the SIS junctions over the $0.8-0.96$ and $0.96-1.12-\mathrm{THz}$ bands, given the frequency-dependent source impedances in Fig. 1. The resulting coupling to the SIS junctions is plotted in Fig. 4 for several combinations of waveguide embedding geometry and SIS device parameters (which are summarized in Tables I and II, respectively).

A number of features are clearly identifiable in these results. First, it is seen that, with a moderate junction current density $\left(J_{c}=8 \mathrm{kA} / \mathrm{cm}^{2}\right)$, the "suspended" and "unsuspended" 0.88-THz designs offer similar coupling efficiencies (50\%-60\% across the $0.8-0.96-\mathrm{THz}$ band, with the "unsuspended" design actually offering slightly higher efficiencies). Moving to a higher current density $\left(J_{c}=15 \mathrm{kA} / \mathrm{cm}^{2}\right)$ significantly improves the coupling to the junctions (due to the junctions' reduced $\omega R C$ product), and the coupling obtained with the "suspended" design is slightly better than that obtained with the 


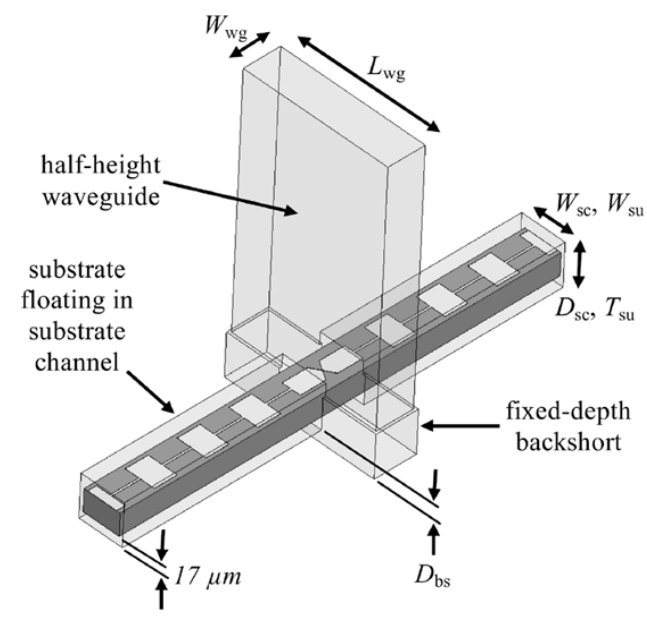

Fig. 2. Suspended substrate waveguide geometry incorporating a half-height waveguide with a bowtie waveguide probe located at the center of the waveguide and a four-section RF choke filter in the substrate channel. The dimensions of the waveguide, substrate channel, and substrate are summarized in Table I. The reference plane at which the embedding impedances are calculated for this structure is located at the center of the bowtie waveguide probe. In other words, this reference plane is located at the input of the on-chip microstrip tuning circuit that is seen in Fig. 3. Ground and dc/IF connections to the SIS device are made by wire-bonding to gold pads at the two ends of the device (beyond the left and right edges of this figure).

TABLE I

WAVEGUIDE MIXER EMBEDDING GEOMETRIES ANALYZED IN FIG. 1

\begin{tabular}{ccccc}
\hline \hline Design & $\begin{array}{c}\text { waveguide } \\
W_{\text {wg }} \times H_{\text {wg }}\end{array}$ & $\begin{array}{c}\text { backshort } \\
\text { depth } \\
D_{\text {bs }}\end{array}$ & $\begin{array}{c}\text { substrate } \\
\text { channel } \\
W_{\text {sc }} \times D_{\mathrm{sc}}\end{array}$ & $\begin{array}{c}\text { substrate }^{\mathrm{b}} \\
W_{\text {su }} \times T_{\text {su }}\end{array}$ \\
\hline $1 \mathrm{THz}$ & $120 \times 240$ & 60 & $90 \times 75$ & $70 \times 40$ \\
$650 \mathrm{GHz}$ & $100 \times 400$ & 80 & $100 \times 75$ & $80 \times 40$ \\
$0.88 \mathrm{THz}$ & $74 \times 296^{\mathrm{a}}$ & 60 & $75 \times 55$ & $60 \times 30$ \\
$0.88 \mathrm{THz}$ & $74 \times 296^{\mathrm{a}}$ & 25 & $90 \times 87$ & $75 \times 45^{\mathrm{c}}$ \\
suspended & $74 \times 250$ \\
\hline \hline
\end{tabular}

all dimensions are given in $\mu \mathrm{m}, W=$ width, $D=$ Depth, $H=$ Height

${ }^{a}$ dimensions used in modeling, actual dimensions are $75 \mu \mathrm{m} \times 300 \mu \mathrm{m}$ and $60 \mu \mathrm{m} \times 240 \mu \mathrm{m}$

${ }^{\mathrm{b}}$ polished fused quartz, $\varepsilon_{\mathrm{r}}=3.8, T_{\mathrm{su}}=$ the substrate thickness after thinning (by polishing)

${ }^{\mathrm{c}}$ suspended by $17 \mu \mathrm{m}$ above the bottom of the substrate channel

"unsuspended" design. Beyond this, Fig. 4(b) demonstrates that the coupling to the junctions in the 1.04- THz design is strongly dependent upon the properties of the NbTiN ground plane- the use of a film deposited at room temperature is expected to result in a strong drop in coupling above $\sim 1 \mathrm{THz}$ (see [9] and [10]), whereas the use of a film deposited at $400{ }^{\circ} \mathrm{C}$ should offer strong coupling over the full $0.96-1.12-\mathrm{THz}$ band (see [11]).

Based upon these calculations, SIS mixers with three combinations of junction current density, NbTiN quality (superconducting transition temperature, $T_{c, \mathrm{NbTiN}}$, and low-temperature normal-state resistivity, $\left.\rho_{\mathrm{n}, 20 \mathrm{~K}}\right)$, and embedding geometry have been produced. Two $0.88-\mathrm{THz}$ mixers (incorporating junctions with $J_{c}=6.5$ and $13 \mathrm{kA} / \mathrm{cm}^{2}, T_{c, \mathrm{NbTiN}}=14.4 \mathrm{~K}$, $\rho_{\mathrm{n}, 20 \mathrm{~K}}=110 \mu \Omega \cdot \mathrm{cm}$ and a suspended embedding geometry) have been produced for Band 3 of the HIFI instrument. Similarly, a 1.04-THz mixer incorporating a suspended-substrate waveguide geometry with $J_{c}=6.5 \mathrm{kA} / \mathrm{cm}^{2}, T_{c, \mathrm{NbTiN}}=16 \mathrm{~K}$, and $\rho_{\mathrm{n}, 20 \mathrm{~K}}=60 \mu \Omega \cdot \mathrm{cm}$ has been produced for Band 4 of the HIFI. In all cases, the SIS junctions are $\mathrm{Nb} / \mathrm{Al}-\mathrm{AlO}_{\mathrm{x}} / \mathrm{Nb}$
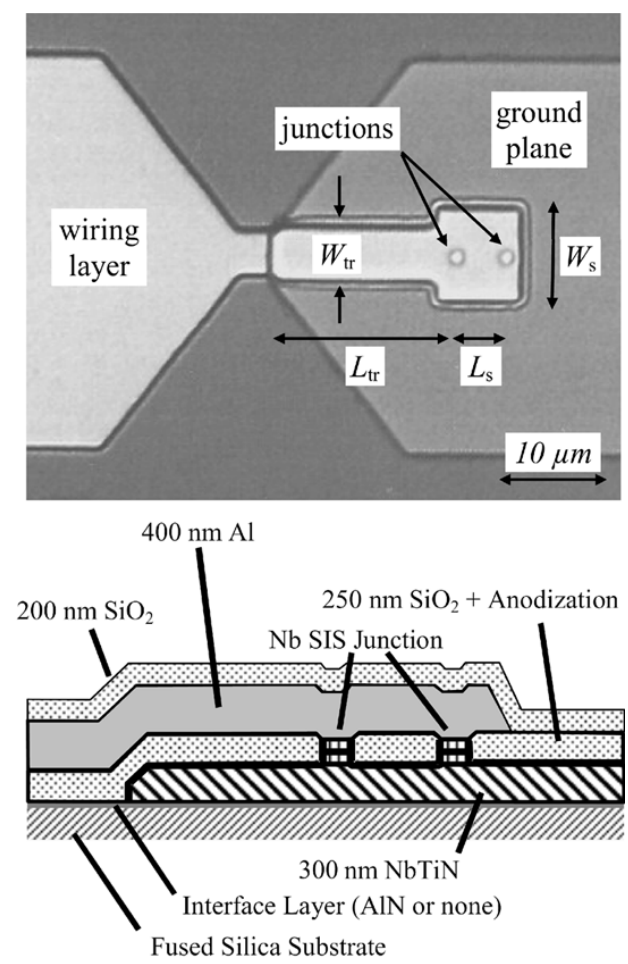

Fig. 3. Twin-junction SIS tuning circuit geometry. Top: photograph of a 1-THz mixer device (adapted from [10]). Note that the dimensions of the microstrip tuning circuit in this image are slightly different than those in the devices used here, but that the basic geometry is the same. Bottom: schematic cross section of the tuning circuit layer structure (adapted from [9]). The dimensions of the microstrip tuning circuit are summarized in Table II.

tunnel junctions with $A \sim 1 \mu \mathrm{m}^{2}$. The microstrip transmission lines combine a $300-\mathrm{nm} \mathrm{NbTiN}$ ground plane, a $250-\mathrm{nm} \mathrm{SiO}_{2}$ dielectric layer (with a nominal $\varepsilon_{r}=3.8$ ), and a $400-\mathrm{nm}$ $\mathrm{Al}$ wiring layer (with a low-temperature dc resistivity of $\left.\rho_{\mathrm{Al}, 4 \mathrm{~K}}=0.3-0.5 \mu \Omega \cdot \mathrm{cm}\right)$.

The fabrication and performance of these mixers are described and discussed further in the following sections.

\section{SIS DEVICE FABRICATION}

The SIS devices used here were produced using a process that is derived from that which was used for the demonstrations of quasi-optical and waveguide mixers incorporating $\mathrm{NbTiN} / \mathrm{SiO}_{2} / \mathrm{Al}$ tuning circuits (see [9] and [10], respectively). However, a number of significant modifications to the process have been made in order to improve the patterning of the SIS junctions and the $\mathrm{Al}$ wiring layer, and to incorporate higher-quality NbTiN ground planes in the 1.04-THz mixers.

The primary modifications to the junction definition process have been: 1) to move from a $\mathrm{CF}_{4}+\mathrm{O}_{2}$ gas mixture to $\mathrm{SF}_{6}+\mathrm{O}_{2}$ for the reactive ion etching of the $\mathrm{Nb}$ junction electrodes (to improve the anisotropy and repeatability of the etch) and 2) to add an $\mathrm{O}_{2}$ plasma etch of the resist pattern following the etch of the top $\mathrm{Nb}$ junction electrode (prior to Ar sputter etching the $\mathrm{Al}-\mathrm{AlO}_{x}$ barrier and reactive ion etching the bottom $\mathrm{Nb}$ electrode). As is discussed in [11], this "resist recessing" step yields a stepped junction profile in which the edges of the active portion of the $\mathrm{Al}-\mathrm{AlO}_{\mathrm{x}}$ barrier are not exposed to the Ar sputter etch of the barrier, since the final size of the top electrode is reached 


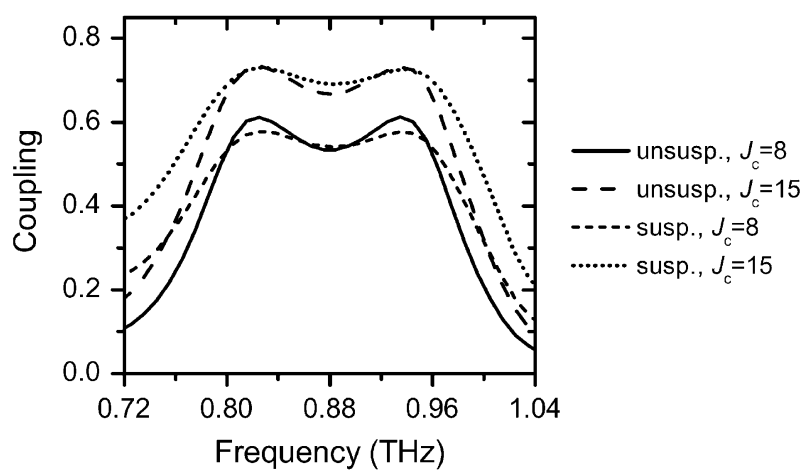

(a)

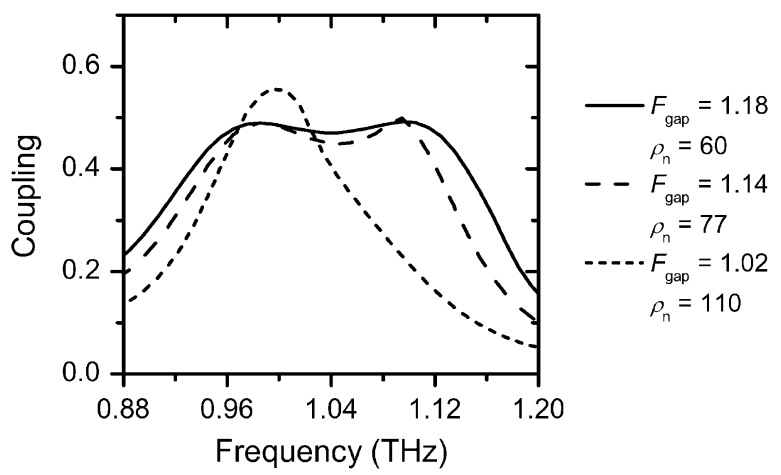

(b) at the completion of the bottom electrode etch. This is expected to improve the quality of a typical junction (i.e., to reduce its leakage current) by reducing the risk of damage to the tunnel barrier during the etch process.

On top of these changes to the junction definition step, the other significant changes to the SIS device process have been: 1) using a chlorine-based reactive ion etch to pattern the $\mathrm{Al}$ wiring layer (which offers improved dimension control relative to the lift-off process that was used in [9] and [10]) and 2) using NbTiN ground planes deposited at $400^{\circ} \mathrm{C}$ (at the Jet Propulsion Laboratory [19]) in the 1.04-THz mixers. Finally, because the lift-off process that was used previously cannot be used to pattern ground planes that are deposited at high temperatures, these films were patterned by reactive ion etching in $\mathrm{SF}_{6}+\mathrm{O}_{2}$.

As in [9] and [10], contact UV lithography is used for all resist pattern definition, RF magnetron sputtering is used to deposit the $\mathrm{SiO}_{2}$ dielectric and passivation layers, and dc magnetron sputtering is used to deposit the $\mathrm{Nb}, \mathrm{NbTiN}$, and Al layers.

Table II summarizes the material characteristics and tuning circuit geometries of the SIS devices that are discussed in the Sections that follow.

\section{DC CURRENT-VOLTAGE CHARACTERISTICS}

Fig. 5 presents the bias current and IF output power as a

Fig. 4. (a) Calculated coupling to the SIS junctions for twin-junction SIS devices mounted in half-height $0.88-\mathrm{THz}$ waveguides with suspended and unsuspended substrates. A slight benefit is obtained from the suspended substrate design if high current-density junctions are used (the values of $J_{c}$ in the legend are given in $\mathrm{kA} / \mathrm{cm}^{2}$ ). (b) Calculated coupling to suspended-substrate twin-junction SIS devices mounted in a half-height $1.04-\mathrm{THz}$ waveguide. A clear dependence of the RF coupling on the superconducting properties of the NbTiN ground plane is observed. Note that the values given the legends are the "gap frequency" and low-temperature normal-state resistivity of NbTiN in-THz and $\mu \Omega \cdot \mathrm{cm}$, respectively $\left(F_{\text {gap, NbTiN }}=2 \Delta_{\mathrm{NbTiN}} / h\right)$.

TABLE II

SIS JUNCTION AND NbTiN GROUND-Plane CHARACTERISTICS AND TUNING Circuit Geometries of the SIS Devices Discussed Here

\begin{tabular}{|c|c|c|c|}
\hline $\begin{array}{l}\text { Wafer Label } \\
\text { Device Number }\end{array}$ & $\begin{array}{c}880 \text { low- } J_{\mathrm{c}} \\
\text { c78-A, c87-A }\end{array}$ & $\begin{array}{c}880 \text { high- } J_{\mathrm{c}} \\
\mathrm{c} 20-\mathrm{B}, \mathrm{c} 78-\mathrm{B}\end{array}$ & $\begin{array}{c}1040 \\
\mathrm{c} 22-\mathrm{C}, \mathrm{f} 49-\mathrm{C}\end{array}$ \\
\hline $\begin{array}{l}\text { Waveguide } \\
\text { Embedding Design } \\
\text { Junction }\end{array}$ & $\begin{array}{c}0.88 \mathrm{THz} \\
\text { suspended }\end{array}$ & $\begin{array}{c}0.88 \mathrm{THz} \\
\text { suspended }\end{array}$ & $\begin{array}{c}1.04 \mathrm{THz} \\
\text { suspended }\end{array}$ \\
\hline$A\left(\mu \mathrm{m}^{2}\right)$ & $0.9,1.1$ & 0.9 & $1.15,1.05$ \\
\hline$J_{\mathrm{c}}\left(\mathrm{kA} / \mathrm{cm}^{2}\right)$ & 6.5 & 13 & 6.5 \\
\hline$R_{2.0 \mathrm{mV}} / \mathrm{R}_{\mathrm{N}}$ & 60 & 20 & $30-50$ \\
\hline \multicolumn{4}{|l|}{ NbTiN } \\
\hline$T_{\text {deposition }}\left({ }^{\circ} \mathrm{C}\right)$ & 20 & 20 & 400 \\
\hline$T_{\mathrm{c}}(\mathrm{K})$ & 14.4 & 14.4 & 16 \\
\hline$\rho_{\mathrm{n}, 20 \mathrm{~K}}(\mu \Omega \cdot \mathrm{cm})$ & 110 & 110 & 60 \\
\hline \multicolumn{4}{|l|}{ Separation } \\
\hline$L_{\mathrm{s}}(\mu \mathrm{m})$ & 5.5 & 5.5 & $5.5,3.5$ \\
\hline$W_{\mathrm{s}}(\mu \mathrm{m})$ & $6-7$ & $6-7$ & $6-7$ \\
\hline \multicolumn{4}{|l|}{ Transformer } \\
\hline$L_{\mathrm{tr}}(\mu \mathrm{m})$ & 24,27 & 24 & 22,20 \\
\hline$W_{\mathrm{tr}}(\mu \mathrm{m})$ & $5-6$ & $5-6$ & 4,5 \\
\hline
\end{tabular}

${ }^{a}$ Note that the first letter/number combination identifies a specific device on the mask layout, while the suffix $(\mathrm{A}, \mathrm{B}, \mathrm{C})$ is used to distinguish between different wafers. Wafer $\mathrm{C}$ uses one mask layout while wafers $\mathrm{A}$ and $\mathrm{B}$ use a common mask design (thus, c78-A and c78-B have the same geometries, other than fabrication tolerances). function of bias voltage for two SIS devices (one $0.88-\mathrm{THz}$ device with $J_{c}=13 \mathrm{kA} / \mathrm{cm}^{2}$ and one $1.04-\mathrm{THz}$ device with $J_{c}=6.5 \mathrm{kA} / \mathrm{cm}^{2}$ ). In general, the junction qualities of these devices, as measured by their subgap to normal-state resistance ratios $\left(R_{2.0 \mathrm{mV}} / R_{N}\right)$, are excellent, with $R_{2.0 \mathrm{mV}} / R_{N}=30-60$ for devices with $J_{c}=6.5 \mathrm{kA} / \mathrm{cm}^{2}$ and $R_{2.0} \mathrm{mV} / R_{N} \sim 20$ for devices with $J_{c}=13 \mathrm{kA} / \mathrm{cm}^{2}$ (at a mixer temperature of 2-2.5 K).

Beyond this, two other features are noted in Fig. 5. First, the photon step in the 1.04-THz device pumped at $1.14 \mathrm{THz}$ is barely wide enough to yield a bias point that is not affected by the Shapiro effect (which can cause instabilities in the IF output power in the output power peaks seen on either side of $V_{\text {Shapiro }}=h F_{\mathrm{LO}} / 2 e=2.1 \mathrm{mV} / \mathrm{THz}$ ). Additionally, whereas the $0.88-\mathrm{THz}$ devices have a typical series resistance in their $\mathrm{Al}$ wiring layer of $0.7 \Omega$ (which is consistent with the low-temperature dc resistivity of the $\mathrm{Al}$ film), the 1.04- $\mathrm{THz}$ devices have a series resistance of $1.5 \Omega$. Note that the resistivity of the Al layer was determined from the resistance of long, narrow strips of $\mathrm{Al}$ deposited under the same conditions as the $\mathrm{Al}$ in the actual devices, while the the series resistance in the $\mathrm{Al}$ wiring of actual junctions is determined from the slope in the junctions' zero-voltage supercurrents. The higher series resistance in the 1.04-THz devices indicates that the resistivity of the $\mathrm{Al}$ wiring layer is higher in the 1.04-THz devices than the $\sigma_{\mathrm{Al}, 4 \mathrm{~K}} \sim$ $0.5 \mu \Omega \cdot \mathrm{cm}$ that is assumed in the design. This is not fully understood, but is thought to be related to the fact that the surface of the $\mathrm{SiO}_{2}$ dielectric layer on which the $\mathrm{Al}$ is deposited is significantly rougher in the $1.04-\mathrm{THz}$ devices than in the $0.88-\mathrm{THz}$ devices (due to differences in the growth mechanics of $\mathrm{SiO}_{2}$ layers on NbTiN films deposited at $400{ }^{\circ} \mathrm{C}$ and room temperature). 


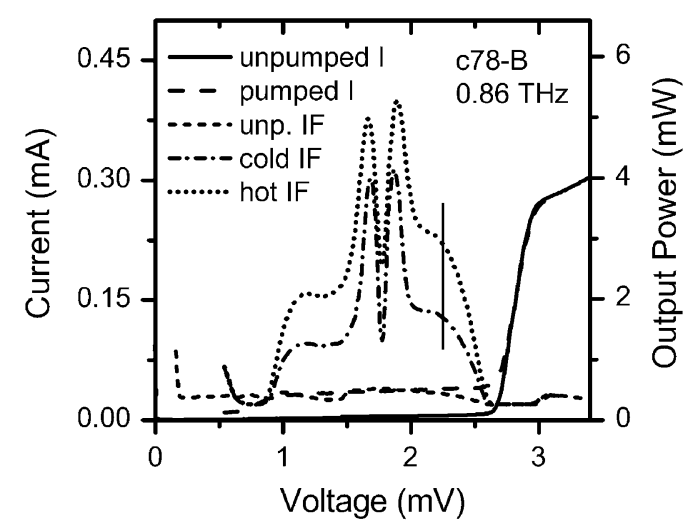

(a)

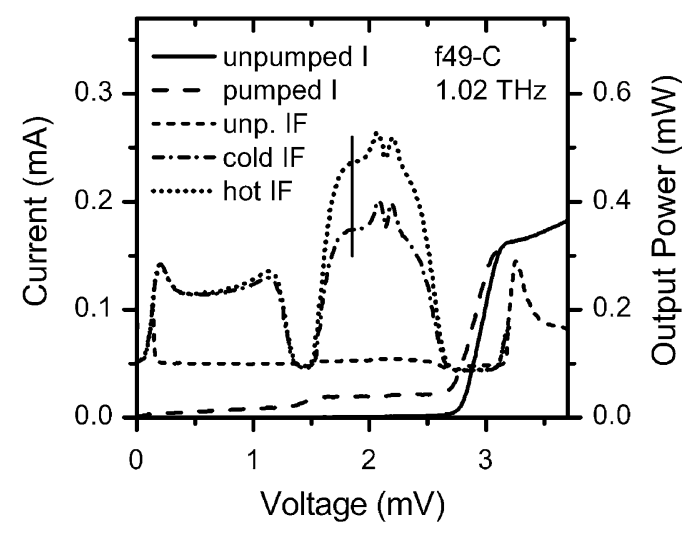

(b)

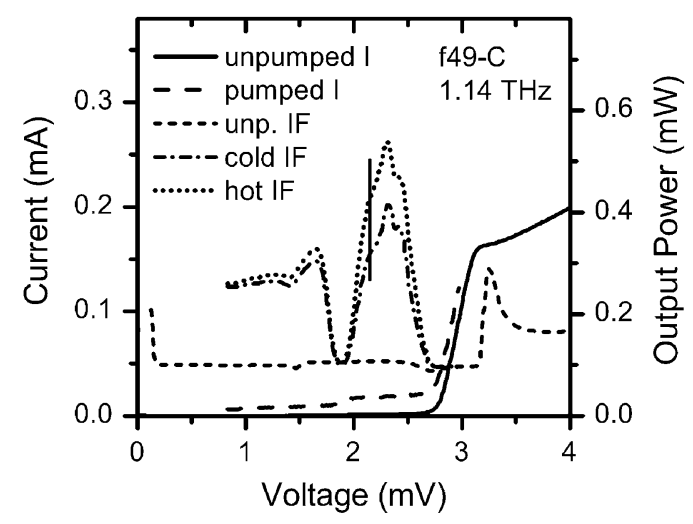

(c)

Fig. 5. (a) Bias current and mixer IF output power as a function of bias voltage for a 0.88 -THz SIS tunnel junction with a current density of $J_{c}=13 \mathrm{kA} / \mathrm{cm}^{2}$, operated at $0.86 \mathrm{THz}$. (b) and (c) Bias current and mixer IF output power as a function of bias voltage for a 1.04-THz SIS junction with $J_{c}=6.5 \mathrm{kA} / \mathrm{cm}^{2}$, operated at $1.02 \mathrm{THz}$ [in (b)] and $1.14 \mathrm{THz}$ [in (c)]. The limited bias range that remains in the $1.04-\mathrm{THz}$ device pumped at $1.14 \mathrm{THz}$ is noted-this is close to the maximum operating frequency for a "traditional" $\mathrm{Nb} / \mathrm{Al}-\mathrm{AlO} \mathrm{x}_{\mathrm{x}} / \mathrm{Nb}$ SIS junction. In both cases, the junction qualities (as measured by their subgap to normal-state resistance ratios) are excellent, with $R_{2.0 \mathrm{mV}} / R_{\mathrm{N}}=30-60$ and 20 obtained for $J_{c}=6.5$ and $13 \mathrm{kA} / \mathrm{cm}^{2}$, respectively. (Note that (b) is adapted from [11].) The vertical markers on the hot and cold IF output power characteristics in each plot indicate the bias voltage that is typically used for RF sensitivity testing - (a) indicates the bias voltage that is used for $0.88-\mathrm{THz}$ mixers, while (b) and (c) indicate the bias voltages that are used for $1.04-\mathrm{THz}$ mixers below 1.04 and above $1.14 \mathrm{THz}$, respectively (no $\mathrm{LO}$ is available in the gap between these ranges).

\section{RF MEASUREMENT SETUP}

The heterodyne sensitivities of the mixers described here have been obtained from conventional $Y$-factor measurements using

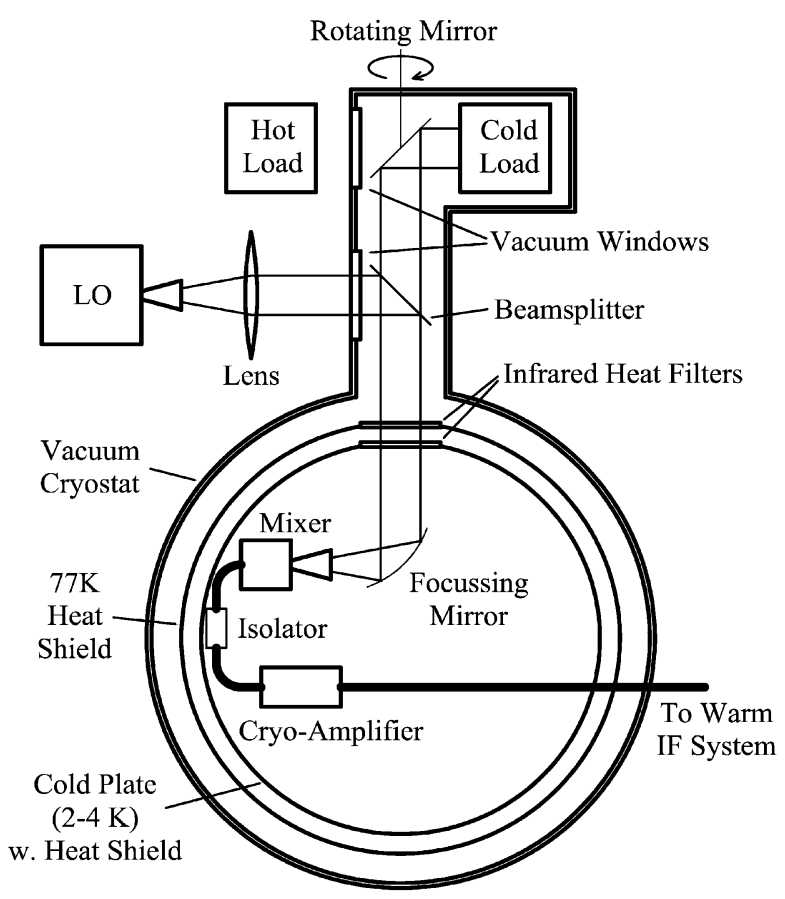

Fig. 6. Schematic representation of the mixer test receiver. This includes a vacuum hot-cold load that is used to improve the accuracy of measurements of the mixer sensitivity by removing the vacuum window from the optical path to the liquid-nitrogen-cooled cold load, and by eliminating the influence of atmospheric absorption of submillimeter radiation on the noise measurements.

a room-temperature "hot" blackbody signal source and a $77 \mathrm{~K}$ "cold" blackbody source (using the Callen-Welton formulation [20] to determine their effective source temperatures from their physical temperatures). Furthermore, because this work is focused on the development of mixers for the HIFI instrument (which includes a window-free, all-reflective optical design), an attempt has been made to minimize the receiver's input coupling losses by: 1) replacing the dielectric focusing lens used previously with a Au-coated mirror on the $4 \mathrm{~K}$ stage of the liquidhelium-cooled test cryostat and 2) making use of a vacuum hot-cold load. In particular, using this vacuum hot-cold load removes the cryostat's vacuum window from the optical path between the mixer and the cold load and eliminates the effect of absorption by atmospheric water vapor, which can be significant at submillimeter wavelengths. Fig. 6 presents a schematic representation of the mixer test system, including the vacuum hot-cold load, in which a rotating mirror located inside the cryostat is used to chop between the hot load (a room-temperature absorber located outside the cryostat) and the cold load. This cold load is produced by coating the bottom of a liquid nitrogen vessel in the receiver's vacuum system with a mixture of silicon carbide grains in black Stycast epoxy [21]. Previous measurements [22] showed that this coating has an emissivity of $\varepsilon \sim 0.95$ at submillimeter wavelengths.

Differences between the mixer test system and the environment of the HIFI instrument have been further minimized by the use of a cryogenic intermediate frequency (IF) amplification system that includes prototypes of the cryogenic $4-8-\mathrm{GHz}$ isolator and low-noise amplifier used in the HIFI instrument (from PamTech, ${ }^{5}$ and the Centro Astronomico de Yebes [23], respec-

\footnotetext{
${ }^{5}$ Passive Microwave Technology Inc., Camarillo, CA.
} 
tively). (The input noise temperature of this IF system is $\sim 8 \mathrm{~K}$, which is only slightly less than the expected $10 \mathrm{~K}$ noise of the IF chain in the HIFI instrument.) Furthermore, the SIS devices have been tested in prototypes of the mixer blocks that will be used in the instrument's 0.88 - and 1.04-THz bands (see [12]), at an operating temperature of $2-2.5 \mathrm{~K}$ (which is close to the expected operating temperature of the mixers in the instrument). (This low operating temperature is reached by pumping on the cryostat's helium bath.)

In reporting the receiver sensitivities presented here, two values are generally given: $T_{\mathrm{N} \text {,rec }}$, the measured double side-band (DSB) receiver sensitivity obtained with the mixer mounted in the test receiver (using a power meter to detect the total output power over the $4-8-\mathrm{GHz}$ IF band), and $T_{\mathrm{N}, \text { mixer+IF }}$, the effective input noise of the mixer and the IF amplifier system (which is obtained by correcting the measured receiver noise temperatures for the calculated/measured losses in the 14- or 49- $\mu \mathrm{m}$ Mylar beamsplitter and the Zitex G104 infrared heat-filters).

Finally, the mixers' direct-detection sensitivities are also presented here because they provide a snapshot of the frequency dependence of the coupling of radiation from an incident optical beam to the SIS junctions. These results have been obtained with a Fourier transform spectrometer in which an evacuated Michelson interferometer is used as a tunable signal source that is injected into the mixer test cryostat via the optical window through which the "hot" signal passes in heterodyne sensitivity measurements. In order to allow the measured results to be compared with the calculated coupling efficiencies, the measured spectra have been corrected for a standing wave in the output of the Michelson interferometer (with a period of $\sim 70 \mathrm{GHz}$ and a peak-to-peak amplitude of $0.8 \mathrm{~dB}$ ) and have been multiplied by a factor of $F$ (in THz) to account for the fact that the measured spectra are proportional to the photon detection efficiency, while the calculated spectra are of the power coupling.

\section{RF MEASUREMENT RESULTS}

Fig. 7 presents the direct-detection sensitivities of several SIS devices in each of the three mixers discussed here (the $0.88-\mathrm{THz}$ mixer with moderate- and high- $J_{c}$ junctions and the $1.04-\mathrm{THz}$ mixer with moderate- $J_{c}$ junctions). From these results, it is seen that both current densities yield efficient coupling to the SIS junctions over broad RF bandwidths. Furthermore, provided that the response is properly centered on the target band (i.e., by properly matching the junction sizes to the tuning circuit geometries), efficient coupling can be obtained over the full 0.8-0.96and $0.96-1.12-\mathrm{THz}$ bands.

Following the direct-detection sensitivities, Fig. 8 presents measured heterodyne sensitivities of several of the SIS devices whose direct-detection sensitivities are plotted in Fig. 7. From these results, it is observed that the $0.88-\mathrm{THz}$ mixers yield $T_{\mathrm{N}, \text { rec }}=300 \mathrm{~K}$ and $T_{\mathrm{N}, \text { mixer }+\mathrm{IF}}=200 \mathrm{~K}$, or better, across a significant fraction of the $0.8-0.96-\mathrm{THz}$ band. The $1.04-\mathrm{THz}$ mixers yield $T_{\mathrm{N}, \text { rec }}=500 \mathrm{~K}$ and $T_{\mathrm{N}, \text { mixer }+\mathrm{IF}}=400 \mathrm{~K}$, or better, across at least $1.03-1.15 \mathrm{THz}$ (for a device tuned to this frequency range). Furthermore, a number of additional observations can be made: 1 ) the sensitivities of the $0.88-\mathrm{THz}$ mixers

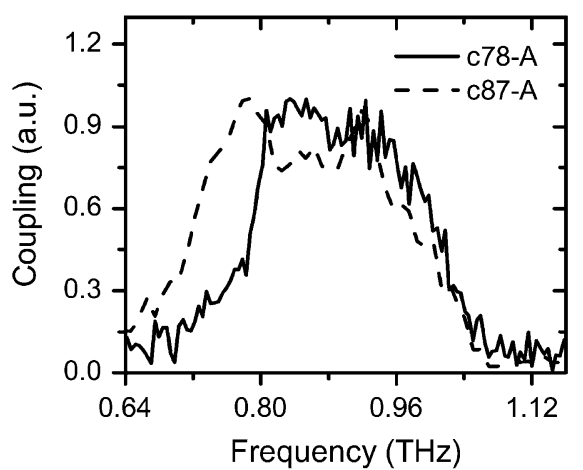

(a)

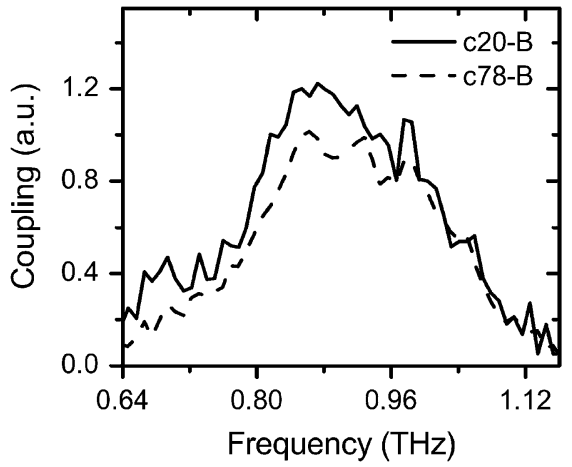

(b)

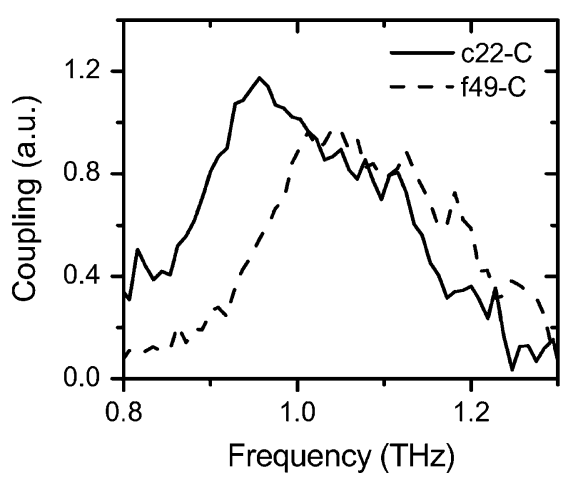

(c)

Fig. 7. Direct-detection sensitivities of several SIS devices mounted in the $0.88-\mathrm{THz}$ and $1.04-\mathrm{THz}$ mixer blocks, biased at $V_{\text {bias }}=2.4-2.5 \mathrm{mV}$. In all cases, the embedding geometry is a half-height waveguide design with the substrate suspended in the substrate channel. (a) Results for $0.88-\mathrm{THz}$ mixers with junction current densities of $J_{c}=6.5 \mathrm{kA} / \mathrm{cm}^{2}$. (b) Results for 0.88 -THz mixers with $J_{c}=13 \mathrm{kA} / \mathrm{cm}^{2}$. (c) Results for $1.04-\mathrm{THz}$ mixers with $J_{c}=6.5 \mathrm{kA} / \mathrm{cm}^{2}$. In each case, strong coupling over a broad RF bandwidth is obtained (although the center-frequency and the frequency dependence of the response is dependent on the tuning circuit geometry and junction size). The geometries of these devices are summarized in Table II, using the device labels identified in the legend of each figure. Note that (c) is adapted from [11].

incorporating 6.5 and $13 \mathrm{kA} / \mathrm{cm}^{2}$ junctions are similar (given the device-to-device variability in their sensitivities); 2) as is discussed further in [11], the use of an NbTiN ground plane deposited at $400{ }^{\circ} \mathrm{C}$ yields sensitive SIS mixers for frequencies up to at least $1.12 \mathrm{THz}$; and 3) the input noise temperatures of the $1.04-\mathrm{THz}$ mixers are $~ 40 \%-50 \%$ higher than those of the 0.88 - THz mixers. Note that receiver sensitivities have not been measured over the full bandwidths of the mxiers, due to a lack of LO power in some frequency bands (especially below $0.86 \mathrm{THz}$ and between $1.04-1.14 \mathrm{THz}$ ). However, the 


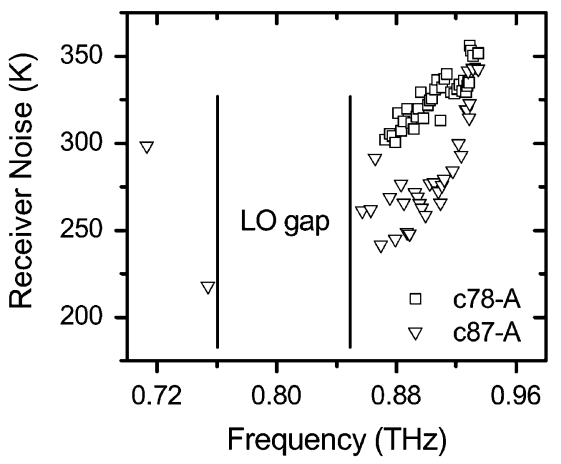

(a)

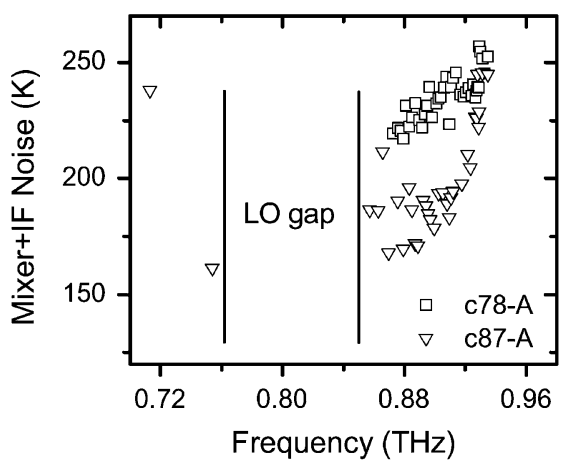

(a)

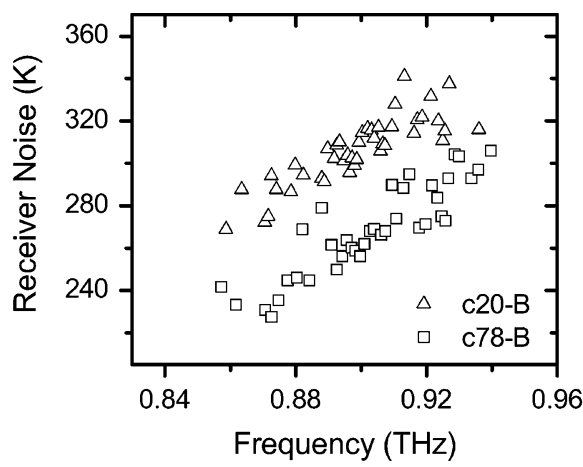

(b)

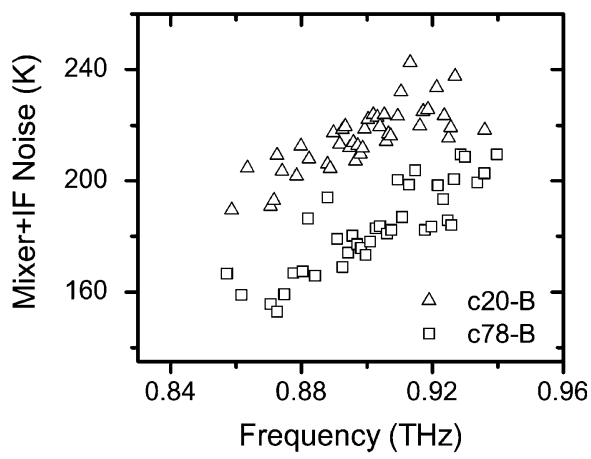

(b)

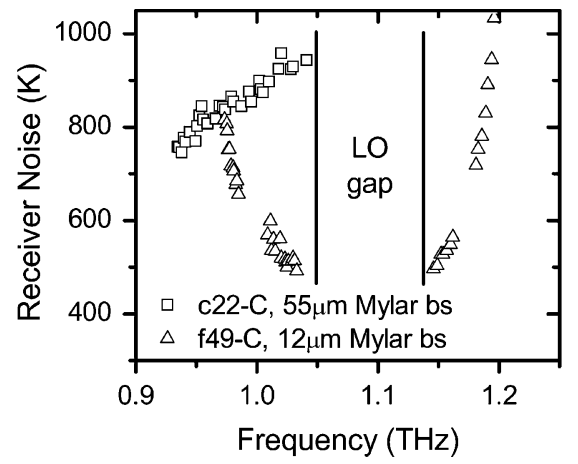

(c)

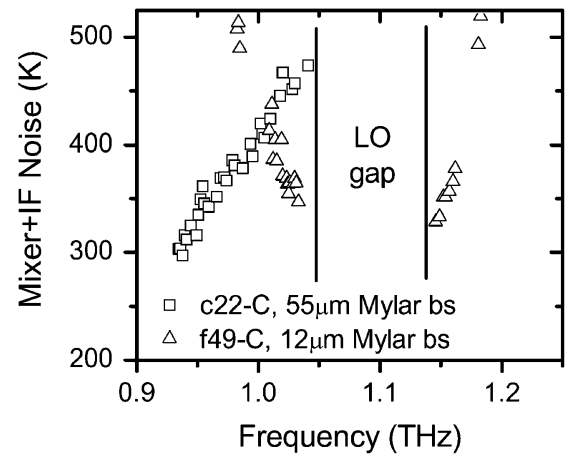

(c)

Fig. 8. Measured DSB receiver noise temperatures $\left(T_{\mathrm{N}, \mathrm{rec}}\right)$ for several of the devices/mixers whose direct detection (FTS) sensitivities are plotted in Fig. 7. At each frequency, the LO power level is adjusted to obtain optimum noise, while the bias voltage is held constant with frequency (at bias voltages that are indicated in Fig. 5). The effective input noise temperatures of the combination of the mixers and IF system $\left(T_{\mathrm{N}, \mathrm{mixer}+\mathrm{IF}}\right)$ (obtained by correcting the measured receiver sensitivities for the calculated/measured losses in the receiver optics), are also shown. All of these measurements are performed at mixer temperatures of 2-2.5 K, averaging over the full bandwidth of a 4-8 GHz IF system with $T_{\mathrm{N}, \mathrm{IF}} \sim 8-10 \mathrm{~K}$. (a) Results for 0.88-THz mixers with $J_{c}=6.5 \mathrm{kA} / \mathrm{cm}^{2}$. (b) Results for 0.88 -THz mixers with $J_{c}=13 \mathrm{kA} / \mathrm{cm}^{2}$. (c) Results for $1.04-\mathrm{THz}$ mixers with $J_{c}=6.5 \mathrm{kA} / \mathrm{cm}^{2}$. In each case, high sensitivity over a broad RF bandwidth is obtained (although the center frequency and the frequency dependence of the response is dependent on the tuning circuit geometry and junction size). The geometries of these devices are summarized in Table II using the device labels indicated in the legends in the bottom right corner of each figure. Note that (c) is adapted from [11]. Note that the LO frequency ranges over which sensitivities are shown are limited by the tuning ranges of the Backward Wave Oscillators that are available. However, the direct detection spectral response curves of these devices, which are seen in Fig. 7, show that the coupling of incident radiation to the junctions over the relevant $0.8-0.96-$ and $0.96-1.12-\mathrm{THz}$ bands is reasonably good.

direct-detection spectral response curves in Fig. 7 show that the coupling of incident radiation to the junctions remains reasonably efficient over the majority of the relevant $0.8-0.96-$ and $0.96-1.12-\mathrm{THz}$ bands.

The determination of the DSB receiver conversion gains for these mixers was complicated by parasitic impedances in the mixers' IF output circuit, which introduced uncertainties to the use of the junction's unpumped shot-noise characteristics to calibrate the noise and gain of the receiver's IF system. However, despite these uncertainties, clear differences could be seen in the gains of the mixers from different wafers-c78-A offered $G_{\text {rec,DSB }} \sim-8 \pm 1 \mathrm{~dB}$ at $0.88 \mathrm{THz}, \mathrm{c} 78$-B offered $G_{\text {rec,DSB }} \sim$ $-4 \pm 1.5 \mathrm{~dB}$ at $0.86 \mathrm{THz}$, and f49-C offered $G_{\text {rec,DSB }} \sim-10 \pm$ $1 \mathrm{~dB}$ at $1.04 \mathrm{THz}$.

The wider RF bandwidths that are expected from the use of higher current-density junctions (see Fig. 4) are not immediately obvious in the measured results in Figs. 7 and 8. However, this may be partly due to the 1-THz "gap frequency" of the NbTiN ground plane limiting the mixers' high-frequency performance (since the devices presented here have been selected to have strong responses in the $0.8-0.96-$ or $0.96-1.12-\mathrm{THz}$ bands, as opposed to maximum bandwidth).

\section{DISCUSSION}

\section{A. Twin-Junction Mixer and Receiver Noise Model}

Calculations of the coupling of radiation to the SIS junctions clearly show that the coupling efficiency increases significantly with increasing current-density (from $\sim 55 \%$ at $J_{c}=$ $8 \mathrm{kA} / \mathrm{cm}^{2}$ to $\sim 70 \%$ at $J_{c}=15 \mathrm{kA} / \mathrm{cm}^{2}$ for the $0.88-\mathrm{THz}$ mixer design). However, measurements of the heterodyne sensitivities of devices with $J_{c}=6.5$ and $13 \mathrm{kA} / \mathrm{cm}^{2}$ show no significant difference in performance. This may be attributed to the fact that the leakage currents of the higher current-density junctions are higher than those of the lower current-density junctions $\left(R_{2.0 \mathrm{mV}} / R_{N} \sim 20\right.$ in place of 30-60). In particular, the larger leakage currents apparently generate sufficient additional shot noise to counteract the improved coupling of radiation to the junctions. (Note that the "sharpness" of the current-voltage characteristics of the lower and higher current-density devices in Fig. 5 is effectively the same, after correcting for the series resistance in their wiring layers- $d V_{\text {gap }} \sim 0.1-0.2 \mathrm{mV}$.)

This conclusion is supported by an analysis of the heterodyne performance of the twin-junction tuning circuit. In this analysis, each of the microstrip transmission-line sections in the RF 
tuning circuit is replaced by a $\pi$-matrix lumped-element circuit model, and the coupling of LO power to the two junctions is then determined in an iterative calculation that takes into account the dependence of the junction admittances on the absorbed LO powers. Given the (different) LO power coupling to the two junctions, the junctions are then replaced by three-port Tucker admittance and noise correlation matrices [24] in order to generate a three-port model for the complete tuning circuit, from which the frequency-dependent mixer noise and gain can be determined. This model of the two-junction tuning circuit is similar to those developed previously in [25] and [26].

In order to evaluate the impact of the junction quality and current density on the mixer noise, the junction model is modified by adding a parallel resistance to the measured currentvoltage characteristic of a junction with $J_{c}=6.5 \mathrm{kA} / \mathrm{cm}^{2}$ and $R_{2.0 \mathrm{mV}} / R_{\mathrm{N}}=60$, and then scaling the voltage and current to obtain the desired gap voltage and junction resistance. (Note that adding this parallel resistance does not change the "sharpness" of the current step and the junction's gap voltage, as is the case in the mixers discussed here.) Finally, the shot noise that is produced by this "excess leakage current" is multiplied by the bias-voltage-dependent factor that was developed in [27] and [28] to account for the amplification of shot noise by multiple Andreev reflection in the leakage current of "leaky" SIS junctions (this factor is $\sim 2.3$ at $V_{\text {bias }}=2.2 \mathrm{mV}$ ).

Receiver noise temperatures are calculated from the calculated mixer noise temperatures and gains by adding input optical losses that are representative of those in the experimental test setup that is being used, plus an IF system with an input noise temperature of $10 \mathrm{~K}$ and a $2 \mathrm{~K}$ physical temperature. A $10 \mathrm{~K}$ IF system noise temperature is used because it is representative of the 4-8-GHz IF system in the HIFI instrument, in which thermal and mechanical constraints have driven the use of a relatively long stainless steel semi-rigid cable between the mixer (at $2 \mathrm{~K}$ ) and the cryoamplifier (at $15 \mathrm{~K}$ ), plus two isolators (one at each end of the long cable).

Fig. 9 presents a comparison of the measured and calculated RF coupling, receiver noise, and receiver conversion gain for two $0.88-\mathrm{THz}$ devices with different junction resistances and tuning circuit dimensions (devices c78-A and c87-A). Because the tuning circuit dimensions and material properties are not known with absolute certainty, the tuning circuit dimensions have been fine-tuned to match the frequency dependence of the calculated RF coupling efficiencies to the measured directdetection sensitivities (applying the same corrections to the geometries of both devices). Furthermore, an additional lossy element is added to the model to fit the calculated noise temperatures to the measured results. For the sake of argument, this excess loss (and the noise that it generates) is represented as a $2.8-\mathrm{dB}$ loss in front of the mixer, at a physical temperature of $2 \mathrm{~K}$. As is seen in Fig. 9, the result is a reasonably consistent match between the measured and calculated receiver noise temperatures for the two mixers. The fact that the calculated receiver conversion gain of c78-A is somewhat lower than the measured gain indicates that at least part of the observed excess receiver noise is likely due to "warm" losses. (Note that the $2.8 \mathrm{~dB}$ of excess loss at $2 \mathrm{~K}$ that is assumed in Fig. 9 can be replaced by $1.1 \mathrm{~dB}$ of excess loss at $300 \mathrm{~K}$.)

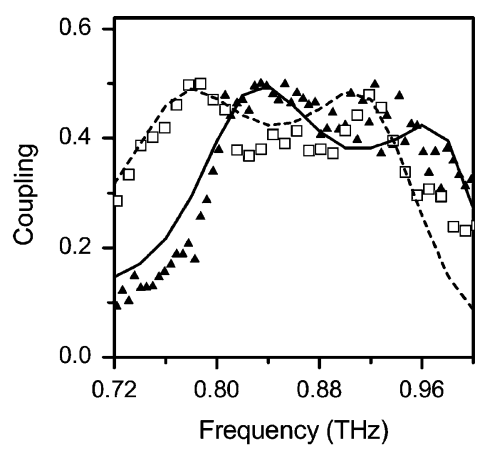

(a)

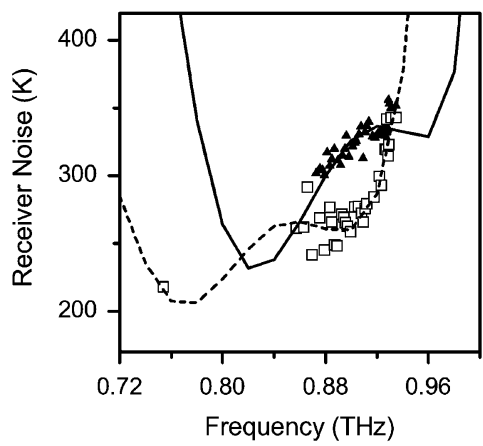

(b)

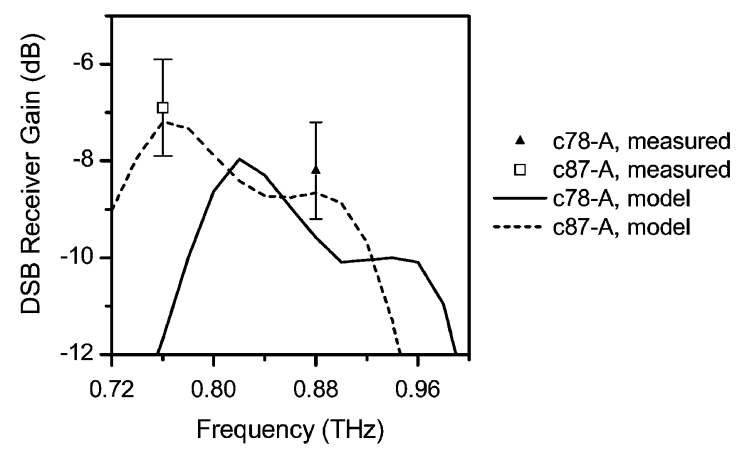

(c)

Fig. 9. Comparison of the measured and calculated direct-detection sensitivities, receiver noise temperatures, and DSB receiver conversion gains for devices c78-A and c87-A from the low- $J_{c} 0.88-\mathrm{THz}$ mixer (with $J_{c}=6.5 \mathrm{kA} / \mathrm{cm}^{2}$ and $R_{2.0 \mathrm{mV}} / R_{\mathrm{N}}=60$ ). Note that the tuning circuit dimensions in the calculations have been tuned slightly to match the frequency dependences of the measured direct-detection sensitivities. Matching the calculated receiver noise temperatures to the measured values requires that an excess loss be inserted in the model (i.e., $2.8 \mathrm{~dB}$ in front of the mixer, at a physical temperature of $2 \mathrm{~K}$ ). The receiver conversion gain values have been corrected for the gain of the receiver's IF system (the error bars in the measured conversion gains stem from uncertainties in estimating this IF system gain).

The calculated excess noise/loss may originate from a number of sources, including the $77 \mathrm{~K}$ blackbody load not being perfectly black (although previous measurements show that it should have an emissivity of $\varepsilon \sim 0.95$ [22]); excess losses in the receiver optics; losses in the corrugated horn, waveguide, and/or substrate channel due to manufacturing errors and/or resistive losses that are not included in the model; dielectric losses in the fused quartz substrate and/or the $\mathrm{SiO}_{2}$ dielectric layer in the tuning circuit, both of which are assumed to be lossless; excess resistive losses in the $\mathrm{Al}$ wiring layer and/or the NbTiN ground plane; resistive losses in the $\mathrm{Nb}$ junction electrodes (which are not taken into account in this model, but 


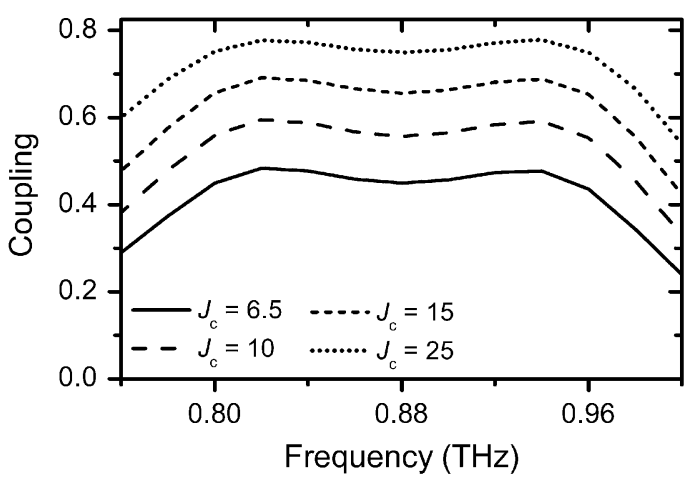

(a)

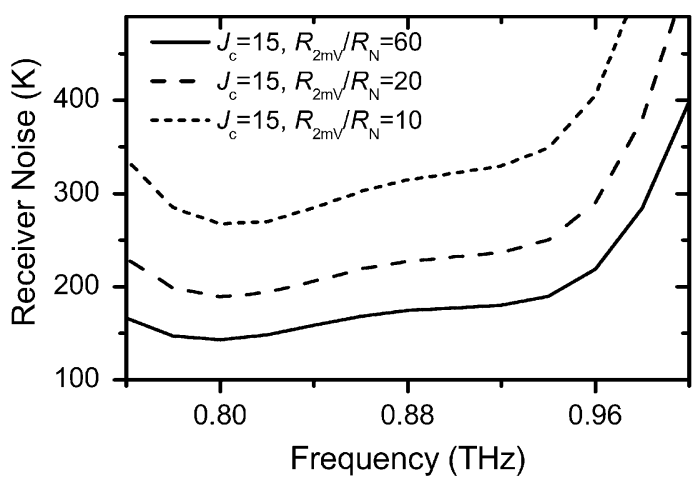

(c)

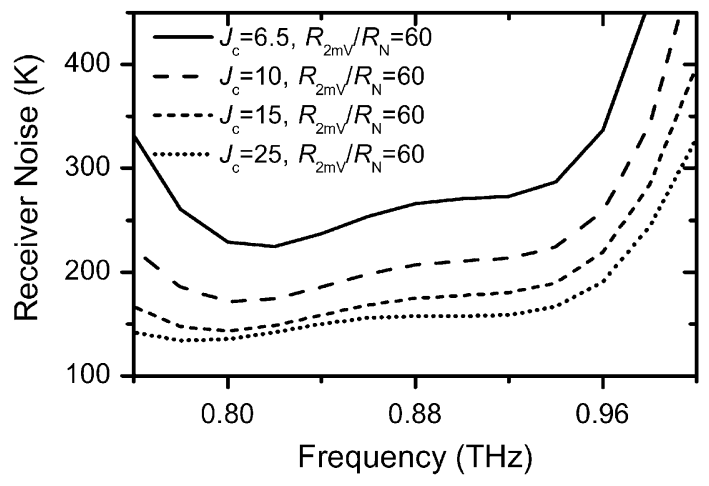

(b)

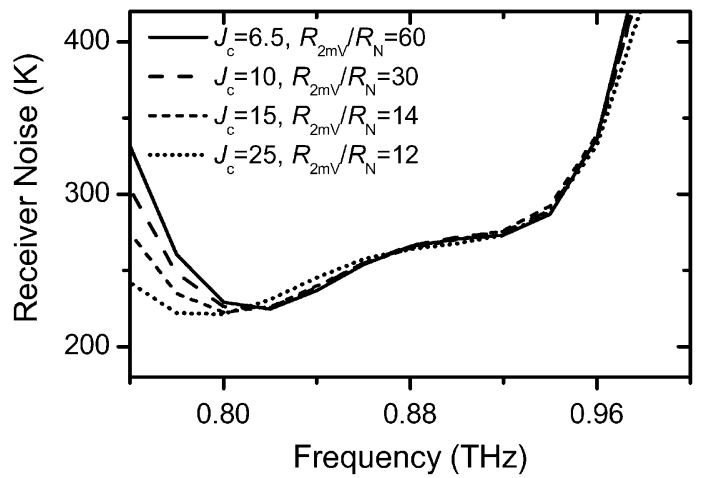

(d)

Fig. 10. Calculated sensitivities of 0.88-THz SIS mixers with twin-junction $\mathrm{NbTiN} / \mathrm{SiO}_{2} / \mathrm{Al}$ tuning circuits and junction current densities ranging from 6.5 to $15 \mathrm{kA} / \mathrm{cm}^{2}$. The same noise and gain contributions for the receiver optics and IF system that were used in Fig. 9 are used in these calculations (including the 2.8 -dB excess loss in front of the mixer). (a) Frequency dependence of the direct-detection coupling to the SIS junctions for different current densities. (b) and (c) Frequency dependence of the DSB receiver noise for different combinations of current density and junction quality $\left(R_{2.0 \mathrm{mV}} / R_{\mathrm{N}}\right)$. (d) Frequency dependence of the DSB receiver noise for different current densities, with the junction quality being defined at each current density as the value that is needed to obtain the same sensitivity as is obtained with $J_{\mathrm{c}}=6.5 \mathrm{kA} / \mathrm{cm}^{2}$ and $R_{2.0 \mathrm{mV}} / R_{\mathrm{N}}=60$. A mixer bias voltage of $2.2 \mathrm{mV}$ is used for all calculations, which is close to the optimum in all cases, while the input noise of the IF system is assumed to be $10 \mathrm{~K}$, which is representative of the 4-8-GHz IF system in the HIFI instrument. For each combination of current density and junction quality, a frequency-independent LO power is used that optimizes the average noise temperature across the $0.8-0.96-\mathrm{THz}$ band.

which are present); and impedance mismatches and/or resistive losses in the mixer's IF circuit (which is represented by a $50 \Omega$ load and an input noise of $10 \mathrm{~K}$ ).

\section{B. Junction Current Density and Quality Versus Receiver Noise}

Using the previously described receiver noise model of the twin-junction mixer, including the $2.8-\mathrm{dB}$ excess loss term that was determined from the results in Fig. 9, the receiver noise of the $0.88-\mathrm{THz}$ mixer has been calculated as a function of junction current density and quality (while adjusting the junction size and the dimensions of the tuning circuit's transformer section to optimize the average RF coupling efficiency over the 0.8-0.96-THz band for each case). The results of these calculations are summarized in Fig. 10.

Note that, for each combination of current density and junction quality, the LO power level has been adjusted to optimize the average sensitivity across the band. (Within the 0.8-0.96-THz band, the sensitivities offered by these "averaged" optimum LO powers do not vary significantly from those that are obtained if the LO power is optimized at each frequency.) The optimum LO power thus increases with both current density and leakage current, although the increase in LO power with current density is driven by a corresponding reduction in junction resistance-the pumping level of the junction, as measured by the alpha parameter $\left(\alpha=e V_{\mathrm{LO}} / h f\right)$, does not change significantly with current density. A bias voltage of $2.2 \mathrm{mV}$ is used for all calculations-this value is close to the optimum in all cases.

Not surprisingly, it is seen that, if the junction quality $\left(R_{2.0 \mathrm{mV}} / R_{\mathrm{N}}\right)$ remains constant, then the receiver noise temperature drops significantly with increasing current density [see Fig. 10(b)]. However, experience shows that junction quality drops with increasing current density in high current density $\left(J_{c}>10-15 \mathrm{kA} / \mathrm{cm}^{2}\right)$ SIS junctions with $\mathrm{AlO}_{\mathrm{x}}$ tunnel barriers. This reduction in junction quality causes a corresponding increase in mixer shot noise [27], [28] that will (partially) offset the improved coupling of incident radiation to the junctions [see Fig. 10(c)]. Indeed, Fig. 10(d) presents the calculated receiver sensitivities for current densities between 6.5 and $15 \mathrm{kA} / \mathrm{cm}^{2}$, with a junction quality in each case that is defined as the minimum value that is needed for junctions with that current density to offer a receiver sensitivity that is equal to that which is obtained with $J_{c}=6.5 \mathrm{kA} / \mathrm{cm}^{2}$ and $R_{2.0 \mathrm{mV}} / R_{\mathrm{N}}=60 \mathrm{kA} / \mathrm{cm}^{2}$.

Reviewing these calculations, it is noted that moving from $J_{c}=6.5$ to 10 and $15 \mathrm{kA} / \mathrm{cm}^{2}$ requires that junction qualities of at least $R_{2.0 \mathrm{mV}} / R_{\mathrm{N}}=30$ and 14 , respectively, are maintained 


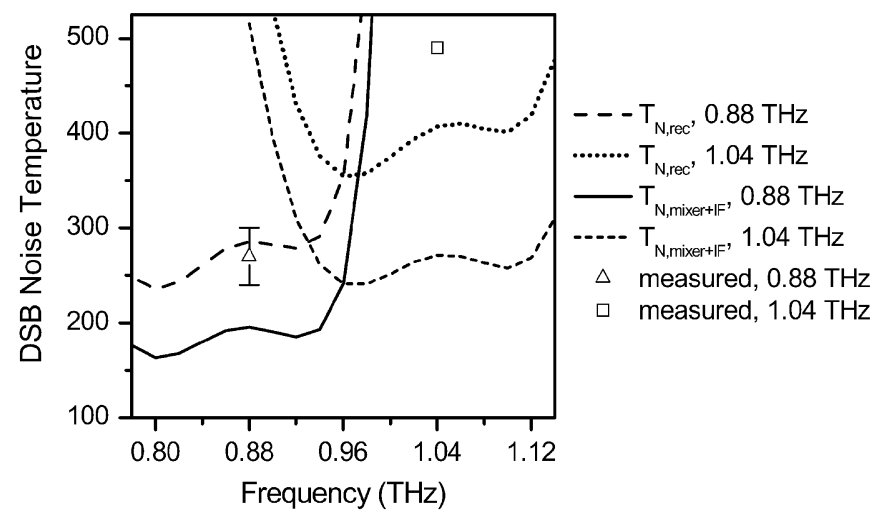

Fig. 11. Calculated receiver and mixer sensitivities $\left(T_{\mathrm{N}, \mathrm{rec}}\right.$ and $\left.T_{\mathrm{N}, \mathrm{mixer}+\mathrm{IF}}\right)$ for 0.88 - and $1.04-\mathrm{THz}$ mixers with current densities of $J_{c}=6.5 \mathrm{kA} / \mathrm{cm}^{2}$ and junction qualities of $R_{2.0 \mathrm{mV}} / R_{\mathrm{N}}=60$. The lower sensitivity of the 1.04-THz mixers is due to a combination of the increased operating frequency (which increases the noise contributions of RF coupling losses and reduces the intrinsic conversion efficiency of the junctions) and the reduced coupling efficiency of the tuning circuit (due to the use of a NbTiN ground plane with a higher conductivity, and thus a smaller penetration depth). (Due to the reduced penetration depth of the NbTiN ground plane deposited at $400{ }^{\circ} \mathrm{C}$, the microstrip sections need to be made longer and narrower to keep the same electrical length and impedance, which increases the series resistance in the Al wiring layer.) For reference, indicators of the measured receiver sensitivities of the 0.88 - and 1.04-THz mixers presented in Fig. 8 are also included.

in order to not lose receiver sensitivity. What is particularly interesting about this result is the fact that it is consistent with the observation that no significant difference is seen in the heterodyne performances of mixers with $J_{c}=6.5$ and $13 \mathrm{kA} / \mathrm{cm}^{2}$ and $R_{2.0 \mathrm{mV}} / R_{\mathrm{N}}=60$ and 20 , respectively. It is also interesting to note that these calculations are also in qualitative agreement with previous observations [29] that $0.64-0.8-\mathrm{THz}$ mixers with $J_{c} \sim 7 \mathrm{kA} / \mathrm{cm}^{2}$ and $R_{2.0 \mathrm{mV}} / R_{\mathrm{N}} \sim 20$ yield similar sensitivities as mixers with $J_{c} \sim 13 \mathrm{kA} / \mathrm{cm}^{2}$ and $R_{2.0 \mathrm{mV}} / R_{\mathrm{N}} \sim 7$.

Note that the increase in the mixers' broad-band sensitivities that is obtained with higher current-density junctions is recognized [see Fig. 10(d)]. However, for the purpose of this comparison, only the sensitivities in the $0.8-0.96-\mathrm{THz}$ range are considered.

\section{Comparison of the 0.88- and 1.04- $\mathrm{THz}$ Results}

This twin-junction mixer sensitivity model can also be used to compare the relative sensitivities of the 0.88 - and $1.04-\mathrm{THz}$ mixers described in this paper. In particular, Fig. 11 presents the calculated sensitivities of 0.88 - and $1.04-\mathrm{THz}$ mixers in which the tuning circuit and junction geometries have been fine-tuned to center the mixers' responses on their respective frequency bands. From this plot, it is seen that a large fraction of the difference in the measured sensitivities of the two mixers is attributable to factors that are included in the previously described mixer noise model. These factors include a drop in the junctions' intrinsic conversion efficiencies and an increase in the noise contributions of optical losses with increasing operating frequency. Additionally, because the penetration depth in NbTiN films deposited at $400{ }^{\circ} \mathrm{C}$ is expected to be smaller than that of films deposited at room temperature (due to their significantly higher normal-state conductivities), the characteristic impedance of a given width of $\mathrm{NbTiN} / \mathrm{SiO}_{2} / \mathrm{Al}$ microstrip transmission line is reduced. As a result, maintaining the impedance and electrical lengths of the microstrip sections in the tuning circuit requires that they be made longer and narrower when replacing a roomtemperature-deposited film with one deposited at $400{ }^{\circ} \mathrm{C}$, which increases the series resistance of the $\mathrm{Al}$ wiring layer. Finally, it is noted that the $1.04-\mathrm{THz}$ devices presented here are characterized by a series resistance in their current-voltage characteristics that is a factor of two higher than that in the $0.88-\mathrm{THz}$ devices. This is a sign that the low-temperature resistivity of the Al wiring layer is likely higher in these devices than the $\rho_{\mathrm{Al}, 4 \mathrm{~K}} \sim 0.5 \mu \Omega \cdot \mathrm{cm}$ that is realized in the $0.88 \mathrm{-THz}$ devices. If this factor-of-two increase in the resistivity of the Al wiring layer is included in the noise model for the 1.04-THz mixer, the calculated receiver noise increases by $\sim 10 \%$ across the band, which brings it much closer to the $T_{\mathrm{N}, \mathrm{rec}}=500 \mathrm{~K}$ that is obtained with device f49-C at 1.04 and $1.14 \mathrm{THz}$.

\section{CONCLUSION}

The development of SIS mixer devices incorporating $\mathrm{NbTiN} / \mathrm{SiO}_{2} / \mathrm{Al}$ microstrip tuning circuits and a parallel pair of "standard" $1-\mu \mathrm{m}^{2} \mathrm{Nb} / \mathrm{Al}-\mathrm{AlO}_{\mathrm{x}} / \mathrm{Nb}$ SIS junctions has enabled the development of low-noise mixers in the $0.8-1.12-\mathrm{THz}$ range. Furthermore, the use of half-height 0.88 - and $1.04-\mathrm{THz}$ waveguide embedding geometries and the optimization of SIS devices to couple efficiently to these embedding circuits has yielded low receiver noise temperatures across the 0.8-0.96and $0.96-1.12-\mathrm{THz}$ bands of the HIFI instrument; a high-resolution heterodyne spectrometer that is being built for the European Space Agency's Herschel Space Observatory.

Analyzing the measured mixer performance with a three-port admittance and noise model of the SIS tuning circuit (in which three-port "Tucker" models of the SIS junctions are combined with lumped-element representations of the microstrip transmission lines), it is found that the frequency dependence of the measured performance is consistent with calculations, but that the measured noise can only be reproduced by inserting $2.8 \mathrm{~dB}$ of excess loss into the noise model (in front of the mixer, at a physical temperature of $2 \mathrm{~K}$ ). The calculated results are also consistent with the observation that mixers containing junctions with current densities of $J_{c}=6.5$ and $13 \mathrm{kA} / \mathrm{cm}^{2}$ yield similar sensitivities. This may be attributed to the fact that the improved RF coupling that is obtained with higher current-density junctions is offset by an increase in junction shot-noise (due to the fact that the higher current-density junctions have larger leakage currents- $R_{2.0 \mathrm{mV}} / R_{\mathrm{N}} \sim 20$ for $J_{c}=13 \mathrm{kA} / \mathrm{cm}^{2}$ versus $R_{2.0 \mathrm{mV}} / R_{\mathrm{N}}=30-60$ for $J_{c}=6.5 \mathrm{kA} / \mathrm{cm}^{2}$ ).

Finally, a significant fraction of the drop in sensitivity of the 1.04- $\mathrm{THz}$ devices relative to the 0.88 - $\mathrm{THz}$ devices is attributed to the intrinsic effects of the higher operating frequency (which causes the conversion gains of the SIS junctions to drop and the noise contributions of RF losses to increase) and the smaller penetration depth in the "higher quality" NbTiN films used in these devices. 


\section{ACKNOWLEDGMENT}

The authors would like to thank W. Laauwen and L. de Jong for performing the mixer measurements described here, M. Eggens, H. Golstein, S. Kikken, D. Nguyen, C. Pieters, H. Schaeffer, and H. Smit for their contributions to the design and construction of the mixers and test systems that were used in this work, and A. Baryshev, J. R. Gao, T. de Graauw, N. Honingh, R. LeDuc, B. Leone, S. Shitov, N. Whyborn, and J. Zmuidzinas for useful discussions.

\section{REFERENCES}

[1] T. de Graauw and F. P. Helmich, "Herschel-HIFI: The heterodyne instrument for the far infrared," in SP-460 The Promise of the Herschel Space Observatory, G. L. Pilbratt, J. Cernicharo, A. M. Heras, T. Prusti, and R. A Harris, Eds. Noordwijk, The Netherlands: ESA Pub. Div., 2001, pp. $45-52$.

[2] G. L. Pilbratt, J. Cernicharo, A. M. Heras, T. Prusti, and R. A Harris, SP-460 The Promise of the Herschel Space Observatory. Noordwijk, The Netherlands: ESA Pub. Div., 2001.

[3] J. W. Kooi, M. Chan, B. Bumble, H. G. LeDuc, P. Schaffer, and T. G. Phillips, "230 and $492 \mathrm{GHz}$ low noise SIS waveguide receivers employing tuned $\mathrm{Nb} / \mathrm{AlO}_{\mathrm{x}} / \mathrm{Nb}$ tunnel junctions," Int. J. Inf. Millim. Waves, vol. 16, pp. 2049-2068, Dec. 1995.

[4] A. Karpov, J. Blondel, M. Voss, and K. H. Gundlach, "A three photon noise SIS heterodyne receiver at submillimeter wavelength," IEEE Trans. Appl. Supercond., vol. 9, no. 6, pp. 4456-4459, Jun. 1999.

[5] C. E. Honingh, S. Haas, D. Hottgenroth, K. Jacobs, and J. Stutzki, "Low noise broadband fixed tuned SIS waveguide mixers at 660 and 800 GHz," IEEE Trans. Appl. Supercond., vol. 7, no. 6, pp. 2582-2586, Jun. 1997.

[6] D. C. Mattis and J. Bardeen, "Theory of the anomalous skin effect in normal and superconducting metals," Phys. Rev., vol. 111, pp. 412-417, 1958.

[7] G. de Lange, J. J. Kuipers, T. M. Klapwijk, R. A. Panhuyzen, H. van de Stadt, and M. W. M. de Graauw, "Superconducting resonator circuits at frequencies above the gap frequency," J. Appl. Phys., vol. 77, pp. 1795-1804, Feb. 1995.

[8] J. Kawamura, J. Chen, D. Miller, J. Kooi, J. Zmuidzinas, B. Bumble, H. G. Leduc, and J. A. Stern, "Low-noise submillimeter-wave NbTiN superconducting tunnel junction mixers," Appl. Phys. Lett., vol. 75, pp. 4013-4015, Dec. 1999.

[9] B. D. Jackson, A. M. Baryshev, G. de Lange, S. V. Shitov, J.-R. Gao, N. N. Iosad, and T. M. Klapwijk, "Low-noise $1 \mathrm{THz}$ superconductorinsulator-superconductor mixer incorporating a $\mathrm{NbTiN} / \mathrm{SiO}_{2} / \mathrm{Al}$ tuning circuit," Appl. Phys. Lett., vol. 79, pp. 436-438, Jul. 2001.

[10] B. D. Jackson, N. N. Iosad, G. de Lange, A. M. Baryshev, W. M. Laauwen, J.-R. Gao, and T. M. Klapwijk, "NbTiN/SiO $/$ /Al tuning circuits for low-noise $1 \mathrm{THz}$ SIS mixers," IEEE Trans. Appl. Supercond., vol. 11 , no. 3, pp. 653-656, Mar. 2001

[11] B. D. Jackson, G. de Lange, T. Zijlstra, M. Kroug, T. M. Klapwijk, and J. A. Stern, "Niobium titanium nitride based superconductor-insulatorsuperconductor mixers for low-noise THz receivers," J. Appl. Phys., vol. 97, no. 11, p. 113904 1-8, Jun. 2005.

[12] G. de Lange, B. Jackson, T. Zijlstra, M. Kroug, and T. M. Klapwijk, "Development of the band 3 and 4 mixer units for HIFI," in Millimeter and Submillimeter Detectors for Astronomy, Proc. of the SPIE, J. Zmuidzinas, W. S. Holland, and S. Withington, Eds. Bellingham, WA: SPIE, 2004, vol. 5498, pp. 268-277.

[13] J. W. Kooi, private communication, 2000-2003.

[14] B. D. Jackson, G. de Lange, W. M. Laauwen, L. de Jong, T. Zijlstra, N. N. Iosad, and T. M. Klapwijk, "THz SIS mixer development for HIFI," in Proc. 13th Int. Symp. Space THz Technol., R. Blundell and E. Tong, Eds. Cambridge, MA, 2002, pp. 561-570.

[15] H. van de Stadt, H. Scheaffer, and L. de Jong, private communication, 1996-1998.
[16] A. M. Baryshev, H. van de Stadt, H. Schaeffer, R. Hesper, T. Zijlstra, M. Zuiddam, W. Wild, and L. de Jong, "Development of a 0.6 THz SIS receiver for ALMA," in Proc. 12th Int. Symp. Space THz Technol., I. Mehdi, Ed.. San Diego, CA, 2001, pp. 581-590.

[17] R. L. Eisenhart and P. J. Khan, "Theoretical and experimental analysis of a waveguide mounting structure," IEEE Trans. Microw. Theory Tech., vol. MTT-19, no. 8, pp. 706-719, Aug. 1971.

[18] C.-Y. E. Tong, R. Blundell, S. Paine, D. C. Papa, J. Kawamura, X. Zhang, J. A. Stern, and H. G. LeDuc, "Design and characterization of a $250-350-\mathrm{GHz}$ fixed-tuned superconductor-insulator-superconductor receiver," IEEE Trans. Microw. Theory Tech., vol. 44, no. 9, pp. 1548-1556, Sep. 1996.

[19] J. A. Stern, B. Bumble, H. G. Leduc, J. W. Kooi, and J. Zmuidzinas, "Fabrication and dc-characterization of NbTiN based SIS mixers for use between 600 and 1200 GHz," in Proc. 9th Int. Symp. Space THz Technol., R. McGrath, Ed.. Pasadena, CA, 1998, pp. 305-313.

[20] H. B. Callen and T. A. Welton, "Irreversibility and generalized noise," Phys. Rev., vol. 83, pp. 34-40, 1951.

[21] T. O. Klaassen, M. C. Diez, J. H. Blok, C. Smorenburg, K. J. Wildeman, and G. Jakob, "Optical characterization of absorbing coatings for submillimeter radiation," in Proc. 12th Int. Symp. Space THz Technol., I. Mehdi, Ed., San Diego, CA, 2001, pp. 400-409.

[22] N. D. Whyborn, private communication, Jul. 2005.

[23] I. Lopez-Fernandez, J. D. G. Puyol, A. B. Cancio, and F. Colomer, "New trends in cryogenic HEMT amplifiers for radio astronomy," presented at the Int. Science and Technology Meeting on the Square Kilometer Array, Berkeley, CA, Jul. 9-13, 2001.

[24] J. R. Tucker and M. J. Feldman, "Quantum detection at millimeter wavelengths," Rev. Mod. Phys., vol. 57, pp. 1055-1113, Oct. 1985.

[25] J. Zmuidzinas, H. G. Leduc, J. A. Stern, and S. R. Cypher, "Two-junction tuning circuits for submillimeter SIS mixers," IEEE Trans. Microw. Theory Tech., vol. 42, no. 4, pp. 698-706, Apr. 1994.

[26] T. Noguchi, S. C. Shi, and J. Inatani, "Parallel connected twin SIS junctions for millimeter and submillimeter-wave mixers-Analysis and experimental verification," IEICE Trans. Elect., vol. E78C, pp. 481-489, May 1995

[27] P. Dieleman, H. G. Bukkems, T. M. Klapwijk, M. Schicke, and K. H. Gundlach, "Observation of Andreev reflection enhanced shot noise," Phys. Rev. Lett., vol. 79, pp. 3486-3489, Nov. 1997.

[28] P. Dieleman and T. M. Klapwijk, "Shot noise beyond the Tucker theory in niobium tunnel junction mixers," Appl. Phys. Lett., vol. 72, pp. 1653-1655, Mar. 1998.

[29] R. Teipen, M. Justen, T. Tils, S. Glenz, C. E. Honingh, K. Jacobs, B. D. Jackson, T. Zijlstra, and M. Kroug, "Influence of junction-quality and current density on HIFI band 2 mixer performance," in Proc. 14th Int. Symp. Space THz Technol., C. Walker and J. Payne, Eds., Tucson, AZ, 2003, pp. 55-62.

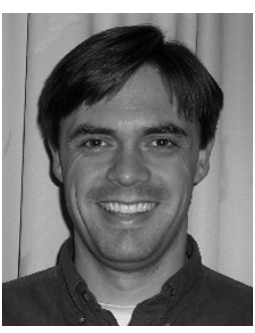

Brian D. Jackson received the B.A.Sc. degree in engineering physics from the University of British Columbia, Vancouver, BC, Canada, in 1995, the M.S. degree in electrical and computer engineering from the University of Toronto, Toronto, ON, Canada, in 1997, and the Ph.D. degree in applied physics from the Delft University of Technology, Delft, The Netherlands, in 2005.

He was a Research Assistant with the National Research Council of Canada, Ottawa, ON, Canada, in 1995, and with the University of Toronto from 1995 to 1997. From 1997 to 1999, he was a Research Engineer with the University of Groningen, Groningen, The Netherlands. Since 1999, he has been an Instrument Scientist with the SRON Netherlands Institute for Space Research, Groningen, The Netherlands. His main research activities are in the areas of SIS junction and mixer development for radio astronomy and system engineering for heterodyne instruments for space- and ground-based telescopes. He has played a role in SIS mixer and/or receiver development for the James Clerk Maxwell Telescope, the Heterodyne Instrument for the Far-Infrared (HIFI) on the European Space Agency's Herschel Space Observatory, and for the Atacama Large Millimeter Array. 


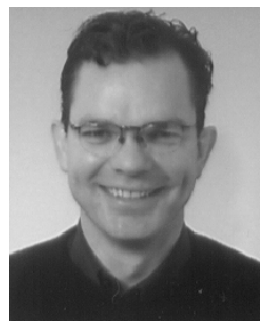

Gert de Lange received the M.S. and Ph.D. degree from the University of Groningen, Groningen, The Netherlands, in 1988 and 1994, respectively, both in applied physics.

From 1994 to 1998, he was a Research Scientist with the Massachussetts Institute of Technology, Cambridge, working on the development of superconducting micromachined heterodyne detector arrays. Since 1998, he has been an Instrument Scientist with the SRON Netherlands Institute for Space Research, Groningen, The Netherlands. His main research activities are in the areas superconducting detector development for low-noise heterodyne receivers for space- or ground-based and airborne submillimeter astronomical instrumentation and the development of space qualified mixer units for the Heterodyne Instrument for the Far-Infrared (HIFI) on the European Space Agency's Herschel Space Observatory.

Tony Zijlstra, photograph and biography not available at the time of publication.

Matthias Kroug, photograph and biography not available at the time of publication.

Jacob W. Kooi, photograph and biography not available at the time of publication.

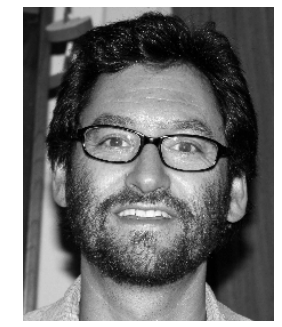

Jeffrey A. Stern received the B.S. degree in physics from Rensselaer Polytechnic Institute, Troy, NY, in 1983, and the Ph.D. degree in applied physics from the California Institute of Technology, Pasadena, in 1991.

Since receiving his doctorate, he has been with the Jet Propulsion Laboratory, Pasadena, most recently as a Senior Member of the Technical Staff. His work has focused on a number of superconducting sensors including $\mathrm{Nb} \mathrm{NbTiN}$ and $\mathrm{NbN}$ SIS mixers, $\mathrm{NbN}$ and NbTiN phononcooled, hot-electron mixers, and, more recently, $\mathrm{NbN}$ single0photon detectors. He was in charge of fabricating, space qualifying, and delivering mixer chips for band 5 of the HIFI instrument on Hershel. He has also been involved in delivering SIS mixer chips to the Caltech Millimeter Array and the Harvard Smithsonian Submillimeter Array. He has also delivered 1.4 THz NbTiN HEB mixers for the Harvard Smithsonian Receiver Lab Telescope in Chile.

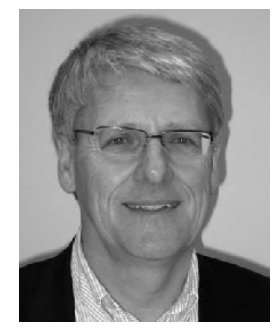

Teun M. Klapwijk received the Ph.D. degree in applied physics from the Delft University of Technology, Delft, The Netherlands, in 1977. His doctoral degree was entitled "Superconducting Microbridges and Radiation Stimulated Superconductivity."

After receiving the doctorate degree, he continued his research with Delft University as an Assistant/Associate Professor, interrupted by periods as a Research Fellow with Harvard University (1979-1980) and as a Summer-Faculty member with the IBM T. J. Watson Research Laboratory, Yorktown Heights, NY (1983). In 1985, he became a Full Professor with the University of Groningen, where he was involved with silicon MOSFETs, superconductor/semiconductor hybrids, and began his long-term collaboration with the SRON Netherlands Institute of Space Research on heterodyne detection with superconducting tunnel junctions and hot-electron bolometers. This research has found its application at the James Clerk Maxwell Telescope, for the Herschel Space Telescope and the Atacama Large Millimeter Array. In 1999, he returned to Delft University, transferring a portion of his ongoing research program and moving into new areas such a superconducting/ferromagnetic hybrids. He is currently a Professor of applied physics, specializing in the field of nanoelectronics, with the Faculty of Applied Sciences, Kavli Institute of Nanoscience, Delft University of Technology, Delft, The Netherlands. He has authored or coauthored over 250 refereed scientific publications.

Prof. Klapwijk has been an elected Fellow of the American Physical Society since 2001. 